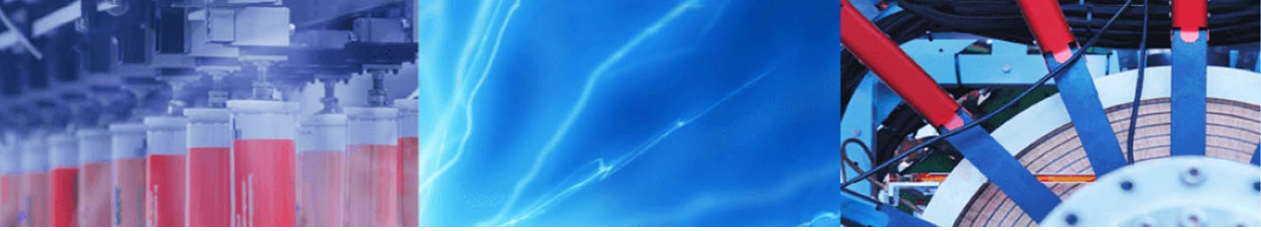

Research Article

\title{
Finding non-linear differential equations and certain identities for the Bernoulli-Euler and Bernoulli-Genocchi numbers
}

\author{
Subuhi Khan ${ }^{1}\left[\right.$ Tabinda Nahid ${ }^{1}[$
}

(c) Springer Nature Switzerland AG 2019

\begin{abstract}
In this research paper, the authors derived the non-linear differential equations for certain hybrid special polynomials related to the Bernoulli polynomials. The families of non-linear differential equations arising from the generating functions of the Bernoulli-Euler and Bernoulli-Genocchi polynomials are derived. Further, these non-linear differential equations are used to derive certain identities and formulas for the Bernoulli-Euler and Bernoulli-Genocchi numbers. However, to provided an exception, a linear differential equation is derived from the generating function of the Genocchi-Euler polynomials.
\end{abstract}

Keywords Bernoulli-Euler numbers · Bernoulli-Genocchi numbers · Genocchi-Euler numbers · Non-linear differential equations

Mathematics Subject Classification 33E30 · 33E20

\section{Introduction}

There has been considerable progress during the recent past on mathematical techniques for studying differential equations that arise in science and engineering. The study of differential equations is a wide field in pure and applied mathematics, physics, and engineering. The problems arising in different areas of science and engineering are usually expressed in terms of differential equations, which in most of the cases have special functions as their solutions. The differential equations and recurrence relations for the Appell polynomials are studied by several authors, see for example $[2,11]$. Recently, the recurrence relations and differential equations for certain hybrid special polynomials related to the Appell sequences are established, see for example $[4,15]$.

During the past 3 decades, the development of nonlinear analysis, dynamical systems and their applications to science and engineering has stimulated renewed enthusiasm for the theory of differential equations. Differential equations play an important role in modeling virtually every physical, technical or biological process ranging from celestial motion to bridge design and to interactions between neurons. Many fundamental laws of physics and chemistry can be formulated in the form of differential equations.

Non-linear differential equations have been extensively used to mathematically model many interesting and important phenomena that are observed in many areas of science and technology. They are inspired by problems which arise in diverse fields such as economics, fluid mechanics, physics, differential geometry, engineering, control theory, material science and quantum mechanics. Recently, the linear and non-linear differential equations from the generating functions of special polynomials and numbers, see for example [5-10]. Kim in [5] initiated a remarkable idea of using non-linear differential equations as a method of obtaining new identities for special polynomials and numbers. This

Subuhi Khan, subuhi2006@gmail.com; Tabinda Nahid, tabindanahid@gmail.com | Department of Mathematics, Aligarh Muslim University, Aligarh, Uttar Pradesh 202002, India.

SN Applied Sciences (2019) 1:217 | https://doi.org/10.1007/s42452-019-0222-0

Received: 31 July 2018 / Accepted: 30 January 2019 / Published online: 6 February 2019 
method turned out to be very useful and it can be applied to many interesting special polynomials and numbers. The recurrence relations and associated differential equations are of fundamental importance in wide variety of fields of pure and applied mathematics, physics and engineering.

A non-linear differential equation is generally more difficult to solve than linear equations. It is common that a nonlinear equation is approximated as linear equation for many practical problems, either in analytical or numerical form. The non-linear nature of the problem is then approximated as series of linear differential equations by simple increment or with correction deviation from the non-linear behaviour. This approach is adopted for the solution of many non-linear engineering problems. Without such procedure most of the non-linear differential equations can not be solved. There are very few methods of solving non-linear differential equations exactly, those that are known typically depend on the equations having particular symmetries.

Sequences of polynomials are a topic of interest in enumerative combinatorics, algebraic combinatorics and applied mathematics. They play an important role in numerous branches of sciences. One of the important classes of polynomial sequences is the class of Appell polynomial sequences [1]. The Appell polynomial sequences appear in different applications in pure and applied mathematics. In 1880, Appell [1] introduced and studied sequences of $n$-degree polynomials $A_{n}(x), n=0,1,2, \ldots$, satisfying the recurrence relation:

$\frac{d}{d x} A_{n}(x)=n A_{n-1}(x), \quad n=1,2, \ldots$

The Appell polynomials are defined by the following generating function:

$A(x, t):=A(t) e^{x t}=\sum_{n=0}^{\infty} A_{n}(x) \frac{t^{n}}{n !}$.
The power series of the analytic function $A(t)$ is given by the following expansion:

$A(t)=A_{0}+\frac{t}{1 !} A_{1}+\frac{t^{2}}{2 !} A_{2}+\cdots+\frac{t^{n}}{n !} A_{n}+\cdots=\sum_{n=0}^{\infty} A_{n} \frac{t^{n}}{n !}, \quad A_{0} \neq 0$,

with $A_{i}(i=0,1,2, \ldots)$ real coefficients.

Based on appropriate selection for the function $A(t)$, different members belonging to the Appell family can be obtained. These members along with their names, generating functions, series definitions and related numbers are given in Table 1.

The Bernoulli, Euler and Genocchi numbers have deep connections with number theory and occur in combinatorics. The Bernoulli numbers enter in many mathematical formulas, such as the Taylor expansion in a neighborhood of the origin of the trigonometric and hyperbolic tangent and cotangent functions and the sums of powers of natural numbers. The Euler numbers are strictly connected with the Bernoulli ones and enter in the Taylor expansion in a neighborhood of the origin of the trigonometric and hyperbolic secant functions. The Genocchi numbers are employed in wide range of applications in number theory, combinatorics, numerical analysis and other fields of applied mathematics. The Genocchi numbers are known to count a large variety of combinatorial objects such as sets of permutations.

In 2013, Subuhi Khan and Raza [3] introduced and studied the 2-iterated Appell polynomials by combining two different sets of Appell polynomials. The 2-iterated Appell polynomials (2IAP) $A_{n}^{[2]}(x)$ are defined by means of the following generating function $[3$, p. $9471(2.5)]$ :

$$
A(t) \mathcal{G}(t) e^{x t}=\sum_{n=0}^{\infty} A_{n}^{[2]}(x) \frac{t^{n}}{n !}
$$

where

Table 1 Certain members belonging to the Appell family

\begin{tabular}{|c|c|c|c|c|}
\hline S. no. & Name of the polynomials and related numbers & $A(t)$ & Generating function & Series definition \\
\hline I. & Bernoulli polynomials and numbers [14] & $\left(\frac{t}{e^{t}-1}\right)$ & $\begin{array}{l}\left(\frac{t}{e^{t}-1}\right) e^{x t}=\sum_{n=0}^{\infty} B_{n}(x) \frac{t^{n}}{n !} \\
\left(\frac{t}{e^{t}-1}\right)=\sum_{n=0}^{\infty} B_{n} \frac{t^{n}}{n !} \\
B_{n}:=B_{n}(0)\end{array}$ & $B_{n}(x)=\sum_{k=0}^{n}\left(\begin{array}{l}n \\
k\end{array}\right) B_{k} x^{n-k}$ \\
\hline II. & Euler polynomials and numbers [14] & $\left(\frac{2}{e^{t}+1}\right)$ & $\begin{array}{l}\left(\frac{2}{e^{t}+1}\right) e^{x t}=\sum_{n=0}^{\infty} E_{n}(x) \frac{t^{n}}{n !} \\
\frac{2}{e^{t}+1}=\sum_{n=0}^{\infty} E_{n} \frac{t^{n}}{n !} \\
E_{n}:=E_{n}(0)\end{array}$ & $E_{n}(x)=\sum_{k=0}^{n}\left(\begin{array}{l}n \\
k\end{array}\right) E_{k} x^{n-k}$ \\
\hline III. & Genocchi polynomials and numbers [13] & $\left(\frac{2 t}{e^{t}+1}\right)$ & $\begin{array}{l}\left(\frac{2 t}{e^{t}+1}\right) e^{x t}=\sum_{n=0}^{\infty} G_{n}(x) \frac{t^{n}}{n !} \\
\frac{2 t}{e^{t}+1}=\sum_{n=0}^{\infty} G_{n} \frac{t^{n}}{n !} \\
G_{n}:=G_{n}(0)\end{array}$ & $G_{n}(x)=\sum_{k=0}^{n}\left(\begin{array}{l}n \\
k\end{array}\right) G_{k} x^{n-k}$ \\
\hline
\end{tabular}




$$
\begin{aligned}
& A(t)=\sum_{n=0}^{\infty} A_{n} \frac{t^{n}}{n !}, \quad A(0) \neq 0, \\
& \mathcal{G}(t)=\sum_{n=0}^{\infty} \mathcal{G}_{n} \frac{t^{n}}{n !}, \quad \mathcal{G}(0) \neq 0 .
\end{aligned}
$$

There exist sequences of numbers $\left\{A_{n}\right\}_{n \geq 0}$ and $\left\{\mathcal{G}_{n}\right\}_{n \geq 0}$, such that the sequence $A_{n}^{[2]}(x)$ satisfies the following relation:

$$
\begin{aligned}
A_{n}^{[2]}(x)= & A_{0} \mathcal{G}_{n}(x)+\left(\begin{array}{c}
n \\
1
\end{array}\right) A_{1} \mathcal{G}_{n-1}(x)+\left(\begin{array}{l}
n \\
2
\end{array}\right) A_{2} \mathcal{G}_{n-2}(x) \\
& +\cdots+A_{n} \mathcal{G}_{0}(x), \quad n=0,1,2, \ldots
\end{aligned}
$$

Based on appropriate selection for the functions $A(t)$ and $\mathcal{G}(t)$, different members belonging to the family of 2-iterated Appell polynomials are obtained. These members along with their names, generating functions, series definitions and related numbers are given in Table 2.

It is given that, if $p_{n}(x)$ and $q_{n}(x)=\sum_{k=0}^{n} q_{n, k} x^{k}$ are sequences of polynomials, then the umbral composition of $q_{n}(x)$ with $p_{n}(x)$ is defined to be the sequence [12]

$q_{n}(p(x))=\sum_{k=0}^{n} q_{n, k} p_{k}(x)$

which is equivalent to condition (1.7).

The set of Appell sequences is closed under the operation of umbral composition of polynomial sequences. Under this operation the set of Appell sequences is an abelian group. Since the generating function of the 2IAP is of the form $A^{\star}(t) e^{x t}$, with $A^{\star}(t)$ as the product of two different functions of $t$. Therefore, the set of all 2IAP sequences also forms an abelian group under the operation of umbral composition. As a consequence of this fact, the Bernoulli-Euler polynomials are equivalent to the Euler-Bernoulli polynomials ${ }_{E} B_{n}(x)$, i.e., ${ }_{B} E_{n}(x) \equiv{ }_{E} B_{n}(x)$. Similarly, the Bernoulli-Genocchi polynomials are equivalent to the Genocchi-Bernoulli polynomials ${ }_{G} B_{n}(x)$, i.e., ${ }_{B} G_{n}(x) \equiv{ }_{G} B_{n}(x)$ and Genocchi-Euler polynomials are equivalent to the Euler-Genocchi polynomials ${ }_{E} G_{n}(x)$, i.e., ${ }_{G} E_{n}(x) \equiv{ }_{E} G_{n}(x)$.

The content of this article are motivated by the work under progress in the direction of obtaining linear and non-linear differential equations involving special polynomials. In this article, the families of non-linear differential equations related to the generating functions of the Bernoulli-Euler and Bernoulli-Genocchi polynomials are constructed. Certain identities for the hybrid special polynomials are established using these non-linear differential equations. To provide an exception, a family of linear differential equations is also derived from the generating function of the Genocchi-Euler polynomials.

\section{Methodologies}

We derive the families of non-linear differential equations from the generating functions of the Bernoulli-Euler polynomials ${ }_{B} E_{n}(x)$ and Bernoulli-Genocchi polynomials ${ }_{B} G_{n}(x)$.

Theorem 2.1 For each $N \in \mathbb{N}$ and $1 \leq i \leq N-1$, the nonlinear differential equation

$$
(N-1) ! H^{N}(t)=(-1)^{N-1} \sum_{j=0}^{N-1} \beta_{j}(N) H^{(j)}(t),
$$

\begin{tabular}{|c|c|c|c|c|}
\hline S. no. & Name of the hybrid polynomials and related numbers & $A(t) ; \mathcal{G}(t)$ & Generating function & Series definition \\
\hline I. & Bernoulli-Euler polynomials and numbers & $\left(\frac{t}{e^{t}-1}\right) ;\left(\frac{2}{e^{t}+1}\right)$ & $\begin{array}{l}\left(\frac{2 t}{e^{2 t}-1}\right) e^{x t}=\sum_{n=0}^{\infty} E_{n}(x) \frac{t^{n}}{n !} \\
\left.\frac{2 t}{e^{2 t}-1}\right)=\sum_{n=0}^{\infty} E_{n} \frac{t^{n}}{n !} \\
{ }_{B} E_{n}:={ }_{B} E_{n}(0)\end{array}$ & $\begin{array}{l}{ }_{B} E_{n}(x)=\sum_{k=0}^{n}\left(\begin{array}{l}n \\
k\end{array}\right) B_{k} E_{n-k}(x) \\
{ }_{B} E_{n}=\sum_{k=0}^{n}\left(\begin{array}{l}n \\
k\end{array}\right) B_{k} E_{n-k}\end{array}$ \\
\hline II. & Bernoulli-Genocchi polynomials and numbers & $\left(\frac{t}{e^{t}-1}\right) ;\left(\frac{2 t}{e^{t}+1}\right)$ & $\begin{array}{l}\left(\frac{2 t^{2}}{e^{2 t}-1}\right) e^{x t}=\sum_{n=0}^{\infty} G_{n}(x) \frac{t^{n}}{n !} \\
\left(\frac{2 t^{2}}{e^{2 t}-1}\right)=\sum_{n=0}^{\infty} G_{n} \frac{t^{n}}{n !} \\
{ }_{B} G_{n}:={ }_{B} G_{n}(0)\end{array}$ & $\begin{array}{l}{ }_{B} G_{n}(x)=\sum_{k=0}^{n}\left(\begin{array}{l}n \\
k\end{array}\right) B_{k} G_{n-k}(x) \\
{ }_{B} G_{n}=\sum_{k=0}^{n}\left(\begin{array}{l}n \\
k\end{array}\right) B_{k} G_{n-k}\end{array}$ \\
\hline III. & Genocchi-Euler polynomials and numbers & $\left(\frac{2 t}{e^{t}+1}\right) ;\left(\frac{2}{e^{t}+1}\right)$ & $\begin{array}{l}\left(\frac{4 t}{\left(e^{t}+1\right)^{2}}\right) e^{x t}=\sum_{n=0}^{\infty} G E_{n}(x) \frac{t^{n}}{n !} \\
\left(\frac{4 t}{\left(e^{t}+1\right)^{2}}\right)=\sum_{n=0}^{\infty} G E_{n} \frac{t^{n}}{n !} \\
{ }_{G} E_{n}:={ }_{G} E_{n}(0)\end{array}$ & $\begin{array}{l}{ }_{G} E_{n}(x)=\sum_{k=0}^{n}\left(\begin{array}{l}n \\
k\end{array}\right) G_{k} E_{n-k}(x) \\
{ }_{G} E_{n}=\sum_{k=0}^{n}\left(\begin{array}{l}n \\
k\end{array}\right) G_{k} E_{n-k}\end{array}$ \\
\hline
\end{tabular}

has a solution $H=H(t)=\frac{2}{e^{2 t}-1}$, where $H^{(j)}(t)=\frac{d^{j} H(t)}{d t^{j}}$, $H^{N}(t)=\underbrace{H(t) \times H(t) \times \cdots \times H(t)}_{N \text {-times }}$ and

Table 2 Certain members belonging to the 2-iterated Appell family 
$\beta_{0}(N+1)=2^{N} N !$,

$\beta_{i}(N+1)=\left(\prod_{n=1}^{i} \sum_{p_{n}=i-n}^{p_{n-1}-1} 2^{p_{n-1}-p_{n}-1}\left(p_{n-1}-1\right)^{<p_{n-1}-p_{n}-1>}\right)$

$\times 2^{p_{i}-1}\left(p_{i}-1\right) !$,

where $p_{0}=N+1 ;(N)^{<n>}=N(N-1) \cdots(N-n+1)$.

Proof Performing differentiation with respect to $t$ on the claimed solution

$H=H(t)=\frac{2}{e^{2 t}-1}$

it follows that

$H^{(1)}=H^{(1)}(t)=(-1)\left(2 H+H^{2}\right)$,

or equivalently

$H^{2}=(-1)\left(2 H+H^{(1)}\right)$.

Again, by taking the derivative with respect to $t$ in the above equation, we find

$2 ! H^{3}=(-1)^{2}\left(8 H+6 H^{(1)}+H^{(2)}\right)$

and consequently

$3 ! H^{4}=(-1)^{3}\left(48 H+44 H^{(1)}+12 H^{(2)}+H^{(3)}\right)$.

Continuing the process up to $N$ times, we get assertion (2.1).

To find the coefficients $\beta_{j}(N)$, let us take the derivatives with respect to $t$ in Eq. (2.1), so that we have

$N ! H^{N+1}=(-1)^{N} 2 N \sum_{j=0}^{N-1} \beta_{j}(N) H^{(j)}+(-1)^{N} \sum_{j=0}^{N-1} \beta_{j}(N) H^{(j+1)}$.

Replacing $N$ by $N+1$ in Eq. (2.1), we have

$N ! H^{N+1}=(-1)^{N} \sum_{j=0}^{N} \beta_{j}(N+1) H^{(j)}$.

Equating the coefficients of $H^{(j)}$ in Eqs. (2.8) and (2.9), we get the following recursive formulas:

$$
\begin{aligned}
& \beta_{0}(N+1)=2 N \beta_{0}(N), \quad \beta_{N}(N+1)=\beta_{N-1}(N), \\
& \beta_{j}(N+1)=2 N \beta_{j}(N)+\beta_{j-1}(N) \quad 1 \leq j \leq N-1 .
\end{aligned}
$$

In view of Eqs. (2.1), (2.4) and (2.5), it follows that

$\beta_{0}(1)=1, \quad \beta_{0}(2)=2$ and $\quad \beta_{1}(2)=1$.

From Eq. (2.10), we have

$\beta_{0}(N+1)=2 N \beta_{0}(N)=2^{2} N(N-1) \beta_{0}(N-1)$

that is

$\beta_{0}(N+1)=2^{N-1} N(N-1)(N-2) \cdots 2 \beta_{0}(2)=2^{N} N !$,

which proves assertion (2.2).

Now, we proceed to find the explicit expressions for the coefficients $\beta_{j}(N)$. From Eq. (2.11), it follows that

$$
\begin{aligned}
\beta_{j}(N+1)= & 2^{2} N(N-1) \beta_{j}(N-1)+2 N \beta_{j-1}(N-1)+\beta_{j-1}(N), \\
= & 2^{3} N(N-1)(N-2) \beta_{j}(N-2)+2^{2} N(N-1) \beta_{j-1}(N-2) \\
& +2 N \beta_{j-1}(N-1)+\beta_{j-1}(N), \\
= & \cdots \\
= & 2^{N-j+1}(N)^{<N-j+1>} \beta_{j-1}(j-1)+\sum_{p_{1}=j}^{N} 2^{N-p_{1}}(N)^{<N-p_{1}>} \beta_{j-1}\left(p_{1}\right) .
\end{aligned}
$$

\section{Consequently}

$\beta_{j}(N+1)=\sum_{p_{1}=j-1}^{N} 2^{N-p_{1}}(N)^{<N-p_{1}>} \beta_{j-1}\left(p_{1}\right)$,

which on again using relation (2.14) in the r.h.s. for $\beta_{j-1}\left(p_{1}\right)$ takes the form

$$
\begin{aligned}
\beta_{j}(N+1)= & \sum_{p_{1}=j-1}^{N} 2^{N-p_{1}}(N)^{<N-p_{1}>} \sum_{p_{2}=j-2}^{p_{1}-1} 2^{p_{1}-1-p_{2}}\left(p_{1}-1\right)^{<p_{1}-1-p_{2}>} \beta_{j-2}\left(p_{2}\right) \\
= & \sum_{p_{1}=j-1}^{N} 2^{N-p_{1}}(N)^{<N-p_{1}>} \sum_{p_{2}=j-2}^{p_{1}-1} 2^{p_{1}-1-p_{2}}\left(p_{1}-1\right)^{<p_{1}-1-p_{2}>} \\
& \times \sum_{p_{3}=j-3}^{p_{2}-1} 2^{p_{2}-1-p_{3}}\left(p_{2}-1\right)^{<p_{2}-1-p_{3}>} \beta_{j-3}\left(p_{3}\right) \\
= & \cdots \\
\beta_{j}(N+1)= & \sum_{p_{1}=j-1}^{N} \sum_{P_{2}=j-2}^{p_{1}-1} \ldots \sum_{p_{n}=j-n}^{p_{n-1}-1} 2^{N-1-p_{n}}(N)^{<N-p_{1}>}\left(p_{1}-1\right)^{<p_{1}-1-p_{2}>} \ldots \\
& \times\left(p_{n-1}-1\right)^{<p_{n-1}-1-p_{n}>} \beta_{j-n}\left(p_{n}\right) .
\end{aligned}
$$

Continuing this process up to $j$ times and using Eq. (2.13), we get assertion (2.3).

The following corollary is an immediate consequence of Theorem 2.1. 
Corollary 2.1 For each $N \in \mathbb{N}$, the non-linear differential equation

$(N-1) ! H^{N}(t, x)=(-1)^{N-1} \sum_{j=0}^{N-1} \beta_{j}(N) H^{(j)}(t, x)$,

has a solution $H=H(t, x)=\frac{2 e^{x t}}{e^{2 t-1}}$, where $\beta_{i}(N), 0 \leq i \leq N-1$ are same as in Eqs. (2.2) and (2.3).

Proof Setting $H^{(j)}(t, x)=H^{(j)}(t) e^{x t} \quad$ a nd $H^{N}(t, x)=\underbrace{H(t) \times H(t) \times \cdots \times H(t)}_{N \text {-times }} e^{x t}$ and multiplying both sides of Eq. (2.1) by $e^{x t}$, we get assertion (2.16).

Theorem 2.2 For each $N \in \mathbb{N}$ and $1 \leq j \leq N-1$, the nonlinear differential equation

$G^{(N)}(t)=(-1)^{N} 2^{N} \sum_{i=1}^{N+1} \alpha_{i-1}(N) G^{i}(t)$,

where $G^{(N)}(t)=\frac{d^{N} G(t)}{d t^{N}}, G^{i}(t)=\underbrace{G(t) \times G(t) \times \cdots \times G(t)}_{i \text {-times }}$ and

$\alpha_{0}(N)=1, \quad \alpha_{N}(N)=N !$,

$\alpha_{j}(N)=j ! \sum_{i_{j}=0}^{N-j} \sum_{i_{j-1}=0}^{N-j-i_{j}} \cdots \sum_{i_{1}=0}^{N-j+1-i_{j}-\cdots-i_{2}}$

$$
(j+1)^{i_{j}}(j-1)^{i_{j-2}} \times \cdots \times(j-(j-2))^{i_{1}},
$$

has a solution $G=G(t)=\frac{1}{e^{2 t}-1}$.

Proof Performing differentiation with respect to $t$ on the claimed solution

$G=G(t)=\frac{1}{e^{2 t}-1}$,

it follows that

$G^{(1)}=G^{(1)}(t)=(-1) 2\left(G+G^{2}\right)$,

Again, by taking the derivative with respect to $t$ in the above equation, we find

$G^{(2)}=(-1)^{2} 2^{2}\left(G+3 G^{2}+2 G^{3}\right)$

and consequently

$G^{(3)}=(-1)^{3} 2^{3}\left(G+7 G^{2}+12 G^{3}+6 G^{4}\right)$.

Continuing the process up to $N$ times, assertion (2.17) follows.
To find the coefficients $\alpha_{i-1}(N)$, let us take the derivatives with respect to $t$ in Eq. (2.17), so that we have

$$
\begin{aligned}
G^{(N+1)}= & (-1)^{N+1} 2^{N+1} \sum_{i=1}^{N+1} i \alpha_{i-1}(N) G^{i} \\
& +(-1)^{N+1} 2^{N+1} \sum_{i=1}^{N+1} i \alpha_{i-1}(N) G^{i+1} .
\end{aligned}
$$

Again, considering Eq. (2.17) with $N$ replaced by $N+1$, we have

$G^{(N+1)}=(-1)^{N+1} 2^{N+1} \sum_{i=1}^{N+2} \alpha_{i-1}(N+1) G^{i}$.

Equating the coefficients of $G^{i}$ in Eqs. (2.24) and (2.25), we find the following recursive formulas:

$\alpha_{0}(N+1)=\alpha_{0}(N), \quad \alpha_{N+1}(N+1)=(N+1) \alpha_{N}(N)$,

$\alpha_{i-1}(N+1)=(i-1) \alpha_{i-2}(N)+i \alpha_{i-1}(N), \quad 2 \leq i \leq N+1$.

In view of Eqs. (2.17), (2.20) and (2.21), it follows that

$\alpha_{0}(0)=1, \quad \alpha_{0}(1)=1 \quad$ and $\quad \alpha_{1}(1)=1$.

From Eq. (2.26), we have

$\alpha_{0}(N+1)=\alpha_{0}(N)=\alpha_{0}(N-1)=\cdots=\alpha_{0}(1)=1$

and

$\alpha_{N+1}(N+1)=(N+1) !$.

Equation (2.29) along with Eq. (2.30), yields assertion (2.18).

Taking $i=2$ in Eq. (2.27), we find

$$
\begin{aligned}
\alpha_{1}(N+1) & =\alpha_{0}(N)+2 \alpha_{1}(N) \\
& =\alpha_{0}(N)+2 \alpha_{0}(N-1)+2^{2} \alpha_{1}(N-1) \\
& =\cdots \\
& =\sum_{i=0}^{N-1} 2^{i} \alpha_{0}(N-i)+2^{N} \alpha_{1}(1)
\end{aligned}
$$

so that

$\alpha_{1}(N+1)=\sum_{i=0}^{N} 2^{i} \alpha_{0}(N-i)$. 
Similarly, for $i=3$ Eq. (2.27) gives

$\alpha_{2}(N+1)=2 \sum_{i=0}^{N-1} 3^{i} \alpha_{1}(N-i)$

consequently for $i=4$, it follows that

$\alpha_{3}(N+1)=3 \sum_{i=0}^{N-2} 4^{i} \alpha_{2}(N-i)$

Proceeding in this way, we deduce that

$\alpha_{k}(N+1)=k \sum_{i_{1}=0}^{N-k+1}(k+1)^{i_{1}} \alpha_{k-1}\left(N-i_{1}\right), \quad 1 \leq k \leq N$.

Now, we proceed to find the explicit expressions for $\alpha_{j}(N)$. In view of Eqs. (2.29), (2.31)-(2.34), we have

$\alpha_{1}(N+1)=\sum_{i_{1}=0}^{N} 2^{i_{1}} \alpha_{0}\left(N-i_{1}\right)=\sum_{i_{1}=0}^{N} 2^{i_{1}}$

$\alpha_{2}(N+1)=2 \sum_{i_{2}=0}^{N-1} 3^{i_{2}} \alpha_{1}\left(N-i_{2}\right)=2 ! \sum_{i_{2}=0}^{N-1} 3^{i_{2}} \sum_{i_{1}=0}^{N-i_{2}-1} 2^{i_{1}}$

$\alpha_{3}(N+1)=3 ! \sum_{i_{3}=0}^{N-2} \sum_{i_{2}=0}^{N-i_{3}-2} \sum_{i_{1}=0}^{N-i_{3}-i_{2}-2} 4^{i_{3}} 3^{i_{2}} 2^{i_{1}}$

Proceeding in the similar manner, we deduce that

$$
\begin{aligned}
\alpha_{j}(N+1)= & j ! \sum_{i_{j}=0}^{N-j+1} \sum_{i_{j-1}=0}^{N-j+1-i_{j}} \times \cdots \times \sum_{i_{1}=0}^{N-j+1-i_{j}-\cdots-i_{2}}(j+1)^{i_{j}}(j)^{i_{j-1}} \\
& \times \cdots \times(j-(j-2))^{i_{1}}, \quad 1 \leq j \leq N
\end{aligned}
$$

which yields assertion (2.19).

The following corollary is an immediate consequence of Theorem 2.2.

Corollary 2.2 For each $N \in \mathbb{N}$, the non-linear differential equation

$G^{(N)}(t, x)=(-1)^{N} 2^{N} \sum_{i=1}^{N+1} \alpha_{i-1}(N) G^{i}(t, x)$, has a solution $G=G(t, x)=\frac{e^{x t}}{e^{2 t}-1}$, where $\alpha_{j}(N), 0 \leq j \leq N$ are same as in Eqs. (2.18) and (2.19).

Proof Setting $G^{(N)}(t, x)=G^{(N)}(t) e^{x t} \quad$ and $G^{i}(t, x)=\underbrace{G(t) \times G(t) \times \cdots \times G(t)}_{\text {imes }} e^{x t}$ and multiplying both sides of Eq. (2.17) by $e^{x t}$, we get assertion (2.39).

In the next section, we establish certain identities arising from the non-linear differential equations obtained above.

\section{Results and summary}

In view of Theorem 2.1, we derive the identities related to the Bernoulli-Euler numbers ${ }_{B} E_{n}$ by proving the following result:

Theorem 3.1 For $n, N \in \mathbb{N}$, the following identities involving Bernoulli-Euler numbers ${ }_{B} E_{n}$ hold true:

${ }_{B} E_{n}^{N}=(-1)^{2 N-n-2} \beta_{N-n-1}(N) \frac{(N-n-1) ! n !}{(N-1) !}, \quad 0 \leq n \leq N-1$

and

${ }_{B} E_{n}^{N}=\sum_{j=0}^{N-1}(-1)^{N-1} N \beta_{j}(N)\left(\begin{array}{c}n \\ N\end{array}\right) \frac{{ }_{B} E_{n-N+j+1}}{(n-N+j+1)}, \quad n \geq N$

where $\beta_{j}(N), 0 \leq j \leq N-1$ are same as in Eqs. (2.2) and (2.3).

Proof In view of Eq. (2.4) and generating equation (Table 2(I)), we have

$H^{(j)}=\left(\frac{d^{j} H(t)}{d t^{j}}\right)=\left(\frac{d^{j}}{d t^{j}}\right)\left(\frac{1}{t} \sum_{n=0}^{\infty}{ }_{B} E_{n} \frac{t^{n}}{n !}\right)$,

which gives

$H^{(j)}=(-1)^{j} j ! t^{-(j+1)}+\sum_{n=0}^{\infty} \frac{{ }_{B} E_{n+j+1}}{(n+j+1)} \frac{t^{n}}{n !}$.

Multiplying both sides of Eq. (3.3) by $t^{N} \sum_{j=0}^{N-1} \beta_{j}(N)$, we find 


$$
\begin{aligned}
t^{N} \sum_{j=0}^{N-1} \beta_{j}(N) H^{(j)}= & \sum_{j=0}^{N-1} \beta_{j}(N)(-1)^{j} j ! t^{N-(j+1)} \\
& +\sum_{j=0}^{N-1} \sum_{n=0}^{\infty} \beta_{j}(N) \frac{{ }_{B} E_{n+j+1}}{(n+j+1)} \frac{t^{n+N}}{n !}
\end{aligned}
$$

which on replacing $j$ by $N-n-1$ in the first term on the r.h.s. becomes

$$
\begin{aligned}
t^{N} \sum_{j=0}^{N-1} \beta_{j}(N) H^{(j)}= & \sum_{n=0}^{N-1}\left(\beta_{N-n-1}(N)(-1)^{N-n-1}(N-n-1) ! n !\right) \frac{t^{n}}{n !} \\
& +\sum_{n=N}^{\infty}\left(\sum_{j=0}^{N-1} \beta_{j}(N) \frac{{ }_{B} E_{n-N+j+1}}{(n-N+j+1)} \frac{n !}{(n-N) !}\right) \frac{t^{n}}{n !} .
\end{aligned}
$$

Again, from Eq. (2.4) and generating function (Table 2(I)), we have

$$
(-1)^{1-N} t^{N}(N-1) ! H^{N}=(-1)^{1-N}(N-1) ! \sum_{n=0}^{\infty}{ }_{B} E_{n}^{N} \frac{t^{n}}{n !} .
$$

In view of Eqs. (2.1), (3.4) and (3.5), we get assertions (3.1) and (3.2).

Corollary 3.1 For $r, n \in \mathbb{N}$, the following explicit formula for the Bernoulli-Euler polynomials ${ }_{B} E_{n}(x)$ of order $r$ holds true:

${ }_{B} E_{n}^{(r)}(x)=\sum_{m_{1}+\cdots+m_{r}+p=n}\left(\begin{array}{c}n \\ m_{1}, m_{2}, \cdots m_{r}, p\end{array}\right){ }_{B} E_{m_{1} B} E_{m_{2}} \cdots{ }_{B} E_{m_{r}} x^{p}$.

Proof From generating function (Table 2(I)), the $n$th Bernoulli-Euler polynomials ${ }_{B} E_{n}(x)$ of order $r$ are defined as:

$\underbrace{\left(\frac{2 t}{e^{2 t}-1}\right) \times\left(\frac{2 t}{e^{2 t}-1}\right) \times \cdots \times\left(\frac{2 t}{e^{2 t}-1}\right)}_{r-\text { times }} e^{x t}=\sum_{n=0}^{\infty}{ }_{B} E_{n}^{(r)}(x) \frac{t^{n}}{n !}$,

which can be written as

$$
\begin{aligned}
& \left(\sum_{m_{1}=0}^{\infty}{ }_{B} E_{m_{1}} \frac{t^{m_{1}}}{m_{1} !}\right)\left(\sum_{m_{2}=0}^{\infty}{ }_{B} E_{m_{2}} \frac{t^{m_{2}}}{m_{2} !}\right) \\
& \quad \ldots\left(\sum_{m_{r}=0}^{\infty}{ }_{B} E_{m_{r}} \frac{t^{m_{r}}}{m_{r} !}\right) \sum_{p=0}^{\infty} x^{p} \frac{t^{p}}{p !}=\sum_{n=0}^{\infty}{ }_{B} E_{n}^{(r)}(x) \frac{t^{n}}{n !} .
\end{aligned}
$$

Applying series rearrangement technique in the I.h.s. of Eq. (3.7), it follows that $\sum_{n=0}^{\infty}\left(\sum_{m_{1}+\cdots+m_{r}+p=n} \frac{{ }_{B} E_{m_{1} B} E_{m_{2}} \cdots{ }_{B} E_{m_{r}}}{m_{1} ! m_{2} ! \cdots m_{r} ! p !} x^{p} n !\right) \frac{t^{n}}{n !}=\sum_{n=0}^{\infty}{ }_{B} E_{n}^{(r)}(x) \frac{t^{n}}{n !}$.

On equating the coefficients of same powers of $t$ in both sides of Eq. (3.8), assertion (3.6) follows.

Next, in view of Theorem 2.2, we derive the identities related to the Bernoulli-Genocchi numbers ${ }_{B} G_{n}$ by proving the following result:

Theorem 3.2 For each $N \in \mathbb{N}$ and $1 \leq j \leq N-1$, the following identity involving Bernoulli-Genocchi numbers ${ }_{B} G_{n}$ hold true:

$$
\begin{aligned}
{ }_{B} G_{n+N+2}= & (-1)^{N}(n+N+2)(n+N+1) \\
& \sum_{i=1}^{N+1} \alpha_{i-1}(N) 2^{N-i+1}{ }_{B} G_{n+2 i}^{i} \frac{n !}{(n+2 i) !}
\end{aligned}
$$

where $\alpha_{i}(N), 0 \leq i \leq N$ are same as in Eqs. (2.18) and (2.19).

Proof From generating function Table 2(II), we have

$\frac{2 t^{2}}{e^{2 t}-1}=\sum_{n=0}^{\infty}{ }_{B} G_{n} \frac{t^{n}}{n !}$

and

$\underbrace{\left(\frac{2 t^{2}}{e^{2 t}-1}\right) \times\left(\frac{2 t^{2}}{e^{2 t}-1}\right) \times \cdots \times\left(\frac{2 t^{2}}{e^{2 t}-1}\right)}_{i \text {-times }}=\sum_{n=0}^{\infty}{ }_{B} G_{n}^{i} \frac{t^{n}}{n !}$.

In view of Eq. (2.20), we find

$$
\begin{aligned}
G^{i}(t) & =\underbrace{\left(\frac{1}{e^{2 t}-1}\right) \times\left(\frac{1}{e^{2 t}-1}\right) \times \cdots \times\left(\frac{1}{e^{2 t}-1}\right)}_{i \text {-times }} \\
& =\frac{1}{\left(2 t^{2}\right)^{i}} \underbrace{\left(\frac{2 t^{2}}{e^{2 t}-1}\right) \times\left(\frac{2 t^{2}}{e^{2 t}-1}\right) \times \cdots \times\left(\frac{2 t^{2}}{e^{2 t}-1}\right)}_{i \text {-times }},
\end{aligned}
$$

which on using Eq. (3.10) becomes

$G^{i}(t)=\frac{1}{2^{i}} \sum_{n=0}^{\infty}{ }_{B} G_{n+2 i}^{i} \frac{n !}{(n+2 i) !} \frac{t^{n}}{n !}$.

Again from Eq. (2.20), we have 
$G(t)=\left(\frac{1}{2 t^{2}}\right)\left(\frac{2 t^{2}}{e^{2 t}-1}\right)=\frac{1}{2 t^{2}} \sum_{n=0}^{\infty}{ }_{B} G_{n} \frac{t^{n}}{n !}$

which gives

$G^{(N)}(t)=\frac{d^{N} G(t)}{d t^{N}}=\frac{1}{2} \sum_{n=0}^{\infty} \frac{{ }_{B} G_{n+N+2}}{(n+N+2)(n+N+1)} \frac{t^{n}}{n !}$

In view of Eqs. (2.17), (3.11) and (3.12), assertion (3.9) follows.

Corollary 3.2 For $r, n \in \mathbb{N}$, the following explicit formula for the Bernoulli-Genocchi polynomials ${ }_{B} G_{n}(x)$ of order $r$ holds true:

${ }_{B} G_{n}^{(r)}(x)=\sum_{m_{1}+\cdots+m_{r}+p=n}\left(\begin{array}{c}n \\ m_{1}, m_{2}, \cdots m_{r}, p\end{array}\right){ }_{B} G_{m_{1} B} G_{m_{2}} \cdots_{B} G_{m_{r}} x^{p}$.

Proof Following the same lines of proof as in Corollary 3.1, assertion (3.13) is proved.

In the next section, a family of linear differential equation from the generating function of the Genocchi-Euler polynomials ${ }_{G} E_{n}(x)$ is derived.

\section{Conclusions}

In the previous section, the non-linear differential equations from the generating functions of the hybrid polynomials related to the Bernoulli, Euler and Genocchi polynomials are considered. However, it is not necessary that every hybrid family yields a non-linear differential equation. To give an example, we consider the Genocchi-Euler polynomials ${ }_{G} E_{n}(x)$.

Let us consider

$F=F(t)=\frac{1}{\left(e^{t}+1\right)^{2}}$.

Performing differentiation with respect to $t$ in Eq. (4.1), it follows that

$F^{(1)}=\frac{-2 e^{t}}{\left(e^{t}+1\right)} F$,

or equivalently

$\left(e^{t}+1\right) F^{(1)}+2 e^{t} F=0$.

Again, by taking the derivatives with respect to $t$ in the above equation, we find

$\left(e^{t}+1\right) F^{(2)}+3 e^{t} F^{(1)}+2 e^{t} F=0$ and consequently

$\left(e^{t}+1\right) F^{(3)}+4 e^{t} F^{(2)}+5 e^{t} F^{(1)}+2 e^{t} F=0$.

Continuing the process up to $N$ times, we get

$\left(e^{t}+1\right) F^{(N)}+\sum_{k=1}^{N} \gamma_{k}(N) e^{t} F^{(k-1)}=0$

Next, to find the coefficients $\gamma_{k}(N)$, let us take the derivatives with respect to $t$ in Eq. (4.5), so that we have

$\left(e^{t}+1\right) F^{(N+1)}+e^{t} F^{(N)}+\sum_{k=1}^{N} \gamma_{k}(N) e^{t} F^{(k-1)}+\sum_{k=1}^{N} \gamma_{k}(N) e^{t} F^{(k)}=0$.

Again, from Eq. (4.5), we have

$\left(e^{t}+1\right) F^{(N+1)}+\sum_{k=1}^{N+1} \gamma_{k}(N+1) e^{t} F^{(k-1)}=0$

Equating the coefficients of $F^{(k)}$ in Eq. (4.6) and (4.7), we find the following recursive formulas:

$\gamma_{1}(N+1)=\gamma_{1}(N), \quad \gamma_{N+1}(N+1)=1+\gamma_{N}(N)$,

$\gamma_{k}(N+1)=\gamma_{k}(N)+\gamma_{k-1}(N), \quad 2 \leq k \leq N$.

In view of Eqs. (4.2), (4.3) and (4.5), it follows that

$\gamma_{1}(1)=2, \quad \gamma_{1}(2)=2$ and $\quad \gamma_{2}(2)=3$.

From Eq. (4.8), we have

$\gamma_{1}(N+1)=\gamma_{1}(N)=\gamma_{1}(N-1)=\cdots=\gamma_{1}(1)=2$,

that is

$\gamma_{1}(N+1)=2$.

Taking $i=2$ in Eq. (4.9), we get

$$
\begin{aligned}
\gamma_{2}(N+1) & =\gamma_{2}(N)+\gamma_{1}(N) \\
& =\gamma_{2}(N-1)+\gamma_{1}(N-1)+\gamma_{1}(N) \\
& =\cdots \\
& =\sum_{i=0}^{N-2} \gamma_{1}(N-i)+\gamma_{2}(2),
\end{aligned}
$$

that is

$\gamma_{2}(N+1)=1+\sum_{i=0}^{N-1} \gamma_{1}(N-i)$.

Similarly, for $i=3$ Eq. (4.9) gives

$\gamma_{3}(N+1)=1+\sum_{i=0}^{N-2} \gamma_{2}(N-i)$.

Proceeding in this way, we deduce that 
$\gamma_{k}(N+1)=1+\sum_{i=0}^{N-k+1} \gamma_{k-1}(N-i), \quad 2 \leq k \leq N$

Therefore, in view of Eqs. (4.5), (4.11) and (4.14), we have the following theorem:

Theorem 4.1 For each $N \in \mathbb{N}$ and $2 \leq k \leq N$, the homogeneous linear differential equation

$$
\begin{aligned}
& \left(e^{t}+1\right) F^{(N)}(t)+\sum_{k=1}^{N} \gamma_{k}(N) e^{t} F^{(k-1)}(t)=0, \\
& \text { where } F^{(N)}(t)=\frac{d^{N} F(t)}{d t^{N}} \text { and } \\
& \gamma_{1}(N+1)=2, \\
& \gamma_{k}(N+1)=1+\sum_{i=0}^{N-k+1} \gamma_{k-1}(N-i),
\end{aligned}
$$

has a solution $F=F(t)=\frac{1}{\left(e^{t}+1\right)^{2}}$.

- The Bernoulli-Euler and Bernoulli-Genocchi polynomials belong to the extended Appell class and form an abelian group under the operation of umbral composition.

- In this paper, the non-linear differential equations for the hybrid members belonging to this extended class are derived.

- The differential equations related to the generating functions of the hybrid special polynomials derived in this article are important from the point of view of their applications in various fields of science.

- It has been demonstrated that it is a captivating idea to use differential equations arising from the generating functions of the hybrid special polynomials to derive enthralling identities related to special polynomials and numbers.

- The approach presented in this article is general and can be extended to other families of hybrid special polynomials.

Acknowledgements This work has been done under Senior Research Fellowship [Award letter No. F./2014-15/NFO-2014-15-OBCUTT-24168/(SA-III/Website)] awarded to the second author by the University Grants Commission, Government of India, New Delhi.

\section{Compliance with ethical standards}

Conflict of interest The authors declare that they have no conflict of interest.

\section{References}

1. Appell P (1880) Sur une classe de polynômes. Ann Sci École Norm Sup 9(2):119-144

2. He MX, Ricci PE (2002) Differential equation of Appell polynomials via the factorization method. J Comput Appl Math 139:231-237

3. Khan S, Raza N (2013) 2-iterated Appell polynomials and related numbers. Appl Math Comput 219(17):9469-9483

4. Khan S, Riyasat M (2016) Differential and integral equations for the 2-iterated Appell polynomials. J Comput Appl Math 306:116-132

5. Kim T (2012) Identities involving Frobenius-Euler polynomials arising from non-linear differential equations. J Number Theory 132(12):2854-2865

6. Kim T, Dolgy DV, Kim DS (2016) Differential equations for Changhee polynomials and their applications. J Nonlinear Sci Appl 9:2857-2864

7. Kim T, Kim DS (2016) A note on nonlinear Changhee differential equations. Russ J Math Phys 23:88-92

8. Kim T, Kim DS (2017) Differential equations associated with Catalan-Daehee numbers and their applications. RACSAM 111:1071-1081

9. Kim DS, Kim T (2015) Some identities for Bernoulli numbers of the second kind arising from a nonlinear differential equation. Bull Korean Math Soc 52:2001-2010

10. Kim S, Kim B-M, Kim J (2016) Differential equations associted with Genocchi polynomials. Global J Pure Appl Math 15(2):4579-4585

11. Özarslan MA, Yilmaz B (2014) A set of finite order differential equations for the Appell polynomials. J Comput Appl Math 259:108-116

12. Roman S (1984) The umbral calculus. Academic Press, New York

13. Sandor J, Crstici B (2004) Handbook of number theory, vol II. Kluwer Academic Publishers, Dordrecht

14. Srivastava HM (2000) Some formulas for the Bernoulli and Euler polynomials at rational arguments. Math Proc Cambr Philos Soc 129:77-84

15. Srivastava HM, Özarslan MA, Yilmaz B (2014) Some families of differential equations associated with the Hermite-based Appell polynomials and other classes of Hermite-based polynomials. Filomat 28(4):695-708

Publisher's Note Springer Nature remains neutral with regard to jurisdictional claims in published maps and institutional affiliations. 